\title{
The NASA Langley Multidisciplinary Uncertainty Quantification Challenge
}

\author{
Luis G. Crespo* \\ National Institute of Aerospace \\ Sean P. Kenny ${ }^{\dagger}$ and Daniel P. Giesy \\ Dynamic Systems and Control Branch, NASA Langley Research Center
}

This paper presents the formulation of an uncertainty quantification challenge problem consisting of five subproblems. These problems focus on key aspects of uncertainty characterization, sensitivity analysis, uncertainty propagation, extreme-case analysis, and robust design.

\section{Introduction}

NASA missions often involve the development of new vehicles and systems that must be designed to operate in harsh domains with a wide array of operating conditions. These missions involve high-consequence and safety-critical systems for which quantitative data is either very sparse or prohibitively expensive to collect. Limited heritage data may exist, but is also usually sparse and may not be directly applicable to the system of interest, making uncertainty quantification extremely challenging. NASA modeling and simulation standards require estimates of uncertainty and descriptions of any processes used to obtain these estimates. The NASA Langley Research Center has developed an uncertainty quantification challenge problem in an effort to focus a community of researchers towards a common problem. This challenge problem features key issues in both uncertainty quantification and robust design using a discipline-independent formulation. While the formulation is indeed discipline-independent, the underlying model, as well as the requirements imposed upon it, describes a realistic aeronautics application. A few high-level details of this application are provided at the end of this document. Additional information is available at: http://uqtools.larc.nasa.gov/nda-uq-challenge-problem-2014/.

\section{Uncertainty Classification}

This challenge problem adopted the generally accepted classifications of uncertainty referred to as aleatory and epistemic [1,2]. Aleatory uncertainty (also called irreducible

${ }^{*}$ Associate Research Fellow, MS 308, NASA LaRC, Hampton VA 23681 USA. AIAA Senior Member.

${ }^{\dagger}$ Senior Research Engineer, MS 308, NASA LaRC, Hampton VA 23681 USA.

${ }^{\ddagger}$ Research Mathematician, MS 308, NASA LaRC, Hampton VA 23681 USA. 
uncertainty, stochastic uncertainty, or variability) is uncertainty due to inherent variation or randomness. Epistemic uncertainty is uncertainty that arises due to a lack of knowledge. Epistemic uncertainty is not an inherent property of the system, but instead it represents the state of knowledge of the parameter and as such it may be reduced if more information is acquired.

According to its physical origin and the system's operating conditions, the value of a parameter can be either fixed (e.g., the mass of a specific element produced by a manufacturing process) or varying (e.g., the mass of any element that can be produced by a manufacturing process). The physical origin of a parameter as well as the knowledge we have about it must be used to create uncertainty models for it. Intervals, fuzzy sets, random variables, probability boxes [3], a.k.a. p-boxes, etc., are different classes of uncertainty models.

While a parameter may be known to be aleatory, sufficient data may not be available to adequately model it as a single random variable. In this case, an approach is to use a random variable with a fixed functional form, e.g., a normal variable but the specific parameters required to fully prescribe it, e.g., the mean and standard deviation, are unknown constants assumed to lie in some given bounded intervals. This results in a distributional p-box, where the physical parameter is indeed an aleatory uncertainty but the parameters prescribing its mathematical model are epistemic uncertainties. A distributional p-box prescribes all the elements of a family of random variables. Conversely, a free $p$-box is defined by prescribed upper and lower bounding cumulative distribution functions and admits any random variable whose cumulative distribution function falls between these bounding functions.

The above considerations lead us to classify each uncertain parameter of the challenge problem as belonging to one the following three categories:

I) An aleatory uncertainty modeled as a random variable with a fixed functional form and known coefficients. This mathematical model is irreducible.

II) An epistemic uncertainty modeled as a fixed but unknown constant that lies within a given interval. This interval is reducible.

III) An aleatory uncertainty modeled as a distributional p-box. Each of the parameters prescribing the random variable is an unknown element of a given interval. These intervals are reducible.

Note that there is no epistemic space associated with a category I parameter since its uncertainty model is fully prescribed. The epistemic space of a category II parameter, which belongs to a family of infinitely many deterministic values, is an interval. The epistemic space of a category III parameter, which belongs to a family of infinitely many probabilistic models, is the Cartesian product of the intervals associated with all the epistemically uncertain parameters of the random variable. Category III parameters may stem from a case where a parameter is known to be random, but there is insufficient data to determine the parameters of its distribution. In this case the epistemic space is given by the confidence intervals of such parameters.

Since most models, especially those used to describe uncertainty, are imperfect; the possibility of improving them always exists. Specifics on what we mean by an "improved" or "reduced" uncertainty model are now in order. Improvements over any given uncertainty model are attained when its epistemic space is reduced. This reduction can be attained by 
performing additional experiments or doing better computational simulations. For instance, denote by $\mathcal{M}_{1}$ a distributional p-box with a Gaussian functional form, mean $\mu \in[a, b]$ and standard deviation $\sigma \in[c, d]$. The uncertainty model $\mathcal{M}_{2}$ is an improvement over $\mathcal{M}_{1}$ if its epistemic space $e_{2}$ satisfies $e_{2} \subset[a, b] \times[c, d]$. Conversely, an irreducible model remains fixed throughout the uncertainty quantification process.

\section{Problem Formulation}

Let $\mathcal{S}$ denote the mathematical model of the multidisciplinary system under investigation. This model is used to evaluate the performance of a physical system and evaluate its suitability. Denote by $\boldsymbol{p}$ a vector of parameters in the system model whose value is uncertain and by $\boldsymbol{d}$ a vector of design variables whose value can be set by the analyst. Furthermore, denote by $\boldsymbol{g}$ a set of requirement metrics used to evaluate the system's performance. The value of $\boldsymbol{g}$ depends on both $\boldsymbol{p}$ and $\boldsymbol{d}$. The system will be regarded as requirement compliant if it satisfies the set of inequality ${ }^{\mathrm{a}}$ constraints $\boldsymbol{g}<\mathbf{0}$. For a fixed value of the design variable $\boldsymbol{d}$, the set of $\boldsymbol{p}$-points where $\boldsymbol{g}<\mathbf{0}$ is called the safe domain, while its complement set is called the failure domain. Therefore, the failure domain corresponding to a fixed design point is comprised of all the parameter points $\boldsymbol{p}$ where at least one of the requirements is violated.

The relationship between the inputs $\boldsymbol{p}$ and $\boldsymbol{d}$, and the output $\boldsymbol{g}$ is given by several functions, each representing a different subsystem or discipline. The function prescribing the output of the multidisciplinary system is given by

$$
\boldsymbol{g}=\boldsymbol{f}(\boldsymbol{x}, \boldsymbol{d}),
$$

where $\boldsymbol{x}$ is a set of intermediate variables whose dependence on $\boldsymbol{p}$ is given by

$$
\boldsymbol{x}=\boldsymbol{h}(\boldsymbol{p}) .
$$

The components of $\boldsymbol{x}$, which can be interpreted as outputs of the fixed discipline analyses in (2), are the inputs to the cross-discipline analyses in (1). The components of $\boldsymbol{g}$ and $\boldsymbol{x}$ are continuous functions of the inputs that prescribe them.

\section{Challenge Overview}

In the following subproblems, initial uncertainty models for the uncertain parameters in vector $\boldsymbol{p}$, as well as software to evaluate Equations (1) and (2), are given (see section V for software availability). We also provide some data, hereafter referred to as "experimental data", which functions as a surrogate for experimental results. These data are exempt from model form uncertainty. An overview of the various tasks of interest is as follows.

- Improve the initial uncertainty models based on the experimental data.

- Decide which uncertainty models should be improved such that the spread in various quantities dependent on $\boldsymbol{p}$ is reduced the most.

- Determine whether various quantities dependent on $\boldsymbol{p}$ are sufficiently insensitive to the uncertainty in any given parameter such that the parameter can be assumed to take on a fixed constant value.




- Determine the range of selected metrics that depend on $\boldsymbol{p}$ for two uncertainty models of $\boldsymbol{p}$. The first uncertainty model is based on information provided in this document. The second model, which is obtained through interaction between the respondents and the challenge problem hosts, is a refinement of the first model. The first and second models differ in the description of a subset of the parameters to be chosen by the respondents. These improved models are based on further experimentation and observation of $\boldsymbol{p}$.

- Identify the particular uncertainty models that yield the extreme values of the ranges mentioned above.

- Determine the design points that provide optimal worst-case probabilistic performance in the presence of uncertainty (optional).

These tasks are at the core of the subproblems presented below. The subproblems, namely uncertainty characterization, sensitivity analysis, uncertainty propagation, extremecase analysis and robust design, are presented in sections A, B, C, D, and E respectively. While the subproblems A, B, C, and D pose analysis tasks for which the value of $\boldsymbol{d}$ is kept fixed at $\boldsymbol{d}=\boldsymbol{d}_{\text {baseline, }}$, subproblem E poses design tasks for which the value of $\boldsymbol{d}$ is to be determined. Specifics on each the subproblems are provided next.

\section{Subproblem A: Uncertainty Characterization}

Here we consider a subsystem of $\mathcal{S}$ whose scalar output $\boldsymbol{x}_{1}$ depends on five uncertain parameters $^{\mathrm{b}}$ as given by

$$
\boldsymbol{x}_{1}=\boldsymbol{h}_{1}\left(\boldsymbol{p}_{1}, \boldsymbol{p}_{2}, \boldsymbol{p}_{3}, \boldsymbol{p}_{4}, \boldsymbol{p}_{5}\right)
$$

Specific information on these parameters is provided in Table 1. The first column provides the parameter's symbol, the second one its category (see above for a description of the categories), and the third one describes its uncertainty model ${ }^{\mathrm{c}}$. While the symbol $\Delta$ denotes the support set or parameter range, $\rho, E[\cdot], V[\cdot]$, and $P[\cdot]$ denote the correlation, expected value, variance, and probability operators respectively. In this subproblem, the tasks of

Table 1. Uncertain parameters.

\begin{tabular}{|c|c|c|}
\hline Symbol & Category & Uncertainty Model \\
\hline $\boldsymbol{p}_{1}$ & III & Unimodal Beta, $3 / 5 \leq E\left[\boldsymbol{p}_{1}\right] \leq 4 / 5,1 / 50 \leq V\left[\boldsymbol{p}_{1}\right] \leq 1 / 25$ \\
\hline $\boldsymbol{p}_{2}$ & II & $\Delta=[0,1]$ \\
\hline $\boldsymbol{p}_{3}$ & I & Uniform, $\Delta=[0,1]$ \\
\hline $\boldsymbol{p}_{4}, \boldsymbol{p}_{5}$ & III & Normal, $-5 \leq E\left[\boldsymbol{p}_{i}\right] \leq 5,1 / 400 \leq V\left[\boldsymbol{p}_{i}\right] \leq 4,|\rho| \leq 1$ for $i=4,5$ \\
\hline
\end{tabular}

interests are as follows:

\footnotetext{
bThe components of vector quantities and vector functions will be specified as subscripts, e.g., the scalar $\boldsymbol{p}_{1}$ is the first component of $\boldsymbol{p}$ while $\boldsymbol{h}_{1}$ is the first function of $\boldsymbol{h}$.

${ }^{\mathrm{c}}$ A random variable whose probability density function (PDF) has a single peak at the interior of the support set will be called unimodal.
} 
A1) Software to evaluate $\boldsymbol{h}_{1}$ and $n=25$ observations of $\boldsymbol{x}_{1}$ corresponding to the "true uncertainty model" are provided, i.e., a model where $\boldsymbol{p}_{1}$ is a fully prescribed Beta random variable, $\boldsymbol{p}_{2}$ is a fixed constant and $\boldsymbol{p}_{4}$ and $\boldsymbol{p}_{5}$ are described by a single and possibly correlated bivariate normal random variable. Use this information to improve the uncertainty model of the category II and III parameters (refer to Section II for the definition of a reduced/improved uncertainty model). The resulting models should only exclude the elements of the original models that fail to explain the observations.

A2) Use an additional $n=25$ observations to validate the models found in A1.

A3) Improve the uncertainty models further by using all the 50 samples available.

A4) Account for the effect of the number of observations $n$ on the fidelity of the resulting uncertainty models. How much better is the model found in A3 as compared to the model found in A1?

\section{Subproblem B: Sensitivity Analysis}

We now consider the multidisciplinary system $\mathcal{S}$ having the input $\boldsymbol{p} \in \mathbb{R}^{21}$ and the output $\boldsymbol{g} \in \mathbb{R}^{8}$. The first 5 input parameters should be modeled using the results from task A3, while the remaining 16 parameters are given in Table 2.

Table 2. Uncertain parameters.

\begin{tabular}{|c|c|c|}
\hline Symbol & Category & Uncertainty Model \\
\hline $\boldsymbol{p}_{6}$ & II & $\Delta=[0,1]$ \\
\hline $\boldsymbol{p}_{7}$ & III & Beta, $0.982 \leq a \leq 3.537,0.619 \leq b \leq 1.080$ \\
\hline $\boldsymbol{p}_{8}$ & III & Beta, $7.450 \leq a \leq 14.093,4.285 \leq b \leq 7.864$ \\
\hline $\boldsymbol{p}_{9}$ & I & Uniform, $\Delta=[0,1]$ \\
\hline $\boldsymbol{p}_{10}$ & III & Beta, $1.520 \leq a \leq 4.513,1.536 \leq b \leq 4.750$ \\
\hline $\boldsymbol{p}_{11}$ & I & Uniform, $\Delta=[0,1]$ \\
\hline $\boldsymbol{p}_{12}$ & II & $\Delta=[0,1]$ \\
\hline $\boldsymbol{p}_{13}$ & III & Beta, $0.412 \leq a \leq 0.737,1.000 \leq b \leq 2.068$ \\
\hline $\boldsymbol{p}_{14}$ & III & Beta, $0.931 \leq a \leq 2.169,1.000 \leq b \leq 2.407$ \\
\hline $\boldsymbol{p}_{15}$ & III & Beta, $5.435 \leq a \leq 7.095,5.287 \leq b \leq 6.945$ \\
\hline $\boldsymbol{p}_{16}$ & II & $\Delta=[0,1]$ \\
\hline $\boldsymbol{p}_{17}$ & III & Beta, $1.060 \leq a \leq 1.662,1.000 \leq b \leq 1.488$ \\
\hline $\boldsymbol{p}_{18}$ & III & Beta, $1.000 \leq a \leq 4.266,0.553 \leq b \leq 1.000$ \\
\hline $\boldsymbol{p}_{19}$ & I & Uniform, $\Delta=[0,1]$ \\
\hline $\boldsymbol{p}_{20}$ & III & Beta, $7.530 \leq a \leq 13.492,4.711 \leq b \leq 8.148$ \\
\hline $\boldsymbol{p}_{21}$ & III & Beta, $0.421 \leq a \leq 1.000,7.772 \leq b \leq 29.621$ \\
\hline
\end{tabular}

The relationship between the input $\boldsymbol{p}$ and the output $\boldsymbol{g}$ is given by Equations (1) and 
(2), where the intermediate variable $\boldsymbol{x} \in \mathbb{R}^{5}$ is given by (3) and

$$
\begin{aligned}
& \boldsymbol{x}_{2}=\boldsymbol{h}_{2}\left(\boldsymbol{p}_{6}, \boldsymbol{p}_{7}, \boldsymbol{p}_{8}, \boldsymbol{p}_{9}, \boldsymbol{p}_{10}\right) \\
& \boldsymbol{x}_{3}=\boldsymbol{h}_{3}\left(\boldsymbol{p}_{11}, \boldsymbol{p}_{12}, \boldsymbol{p}_{13}, \boldsymbol{p}_{14}, \boldsymbol{p}_{15}\right), \\
& \boldsymbol{x}_{4}=\boldsymbol{h}_{4}\left(\boldsymbol{p}_{16}, \boldsymbol{p}_{17}, \boldsymbol{p}_{18}, \boldsymbol{p}_{19}, \boldsymbol{p}_{20}\right) \\
& \boldsymbol{x}_{5}=\boldsymbol{p}_{21}
\end{aligned}
$$

Note that the propagation of the uncertainty model of $\boldsymbol{p}$ through $\boldsymbol{h}$ yields distributional p-boxes for $\boldsymbol{x}_{1}, \boldsymbol{x}_{2}, \boldsymbol{x}_{3}$ and $\boldsymbol{x}_{4}$. If the uncertainty models of the category II and III parameters are improved, so will be the resulting p-boxes of $\boldsymbol{x}_{1}, \boldsymbol{x}_{2}, \boldsymbol{x}_{3}$ and $\boldsymbol{x}_{4}$.

In this subproblem we want to perform the following tasks:

B1) Rank the 4 category II-III parameters entering Equation (3) according to degree of refinement in the p-box of $\boldsymbol{x}_{1}$ which one could hope to obtain by refining their uncertainty models. Are there any parameters that can be assumed to take on a fixed constant value without incurring a significant error? If so, evaluate/bound this error, list which parameters and set their corresponding constant values. Do the same for the 4 category II-III parameters prescribing $\boldsymbol{x}_{2}, \boldsymbol{x}_{3}$ and $\boldsymbol{x}_{4}$.

B2) Rank the 17 category II-III parameters of Tables 1 and 2 according to the reduction in the range of the expected value

$$
J_{1}=E\left[w\left(\boldsymbol{p}, \boldsymbol{d}_{\text {baseline }}\right)\right],
$$

which one could hope to obtain by refining their uncertainty models. In this expression,

$$
w(\boldsymbol{p}, \boldsymbol{d})=\max _{1 \leq i \leq 8} \boldsymbol{g}_{i}=\max _{1 \leq i \leq 8} \boldsymbol{f}_{i}(\boldsymbol{h}(\boldsymbol{p}), \boldsymbol{d}),
$$

is the worst-case requirement metric. Are there any parameters that can be assumed to take on a fixed constant value without incurring a significant error? If so, evaluate/bound this error, list which parameters and set their corresponding constant values.

B3) Rank the 17 category II-III parameters of Tables 1 and 2 according to the reduction in the range of the failure probability:

$$
J_{2}=1-P\left[w\left(\boldsymbol{p}, \boldsymbol{d}_{\text {baseline }}\right)<0\right],
$$

which one could hope to obtain by refining their uncertainty models ${ }^{\mathrm{d}}$. Are there any parameters that can be assumed to take on a fixed constant value without incurring significant error? If so, evaluate/bound this error, list which parameters and set their corresponding constant values.

Compare the above rankings and eventual parameter simplifications. Note that while the tasks in B1 are of interest to experts in the disciplines modelled by $\boldsymbol{h}$, the tasks in B2 and B3 are of interest to analysts of the integrated system. Further notice that each ranking can be used to determine the key parameters whose uncertainty model we want to improve.

\footnotetext{
dote that $J_{2}$ is equal to $P\left[\bigcup_{i=1}^{8}\left\{\boldsymbol{p}: \boldsymbol{f}_{i}\left(\boldsymbol{h}(\boldsymbol{p}), \boldsymbol{d}_{\text {baseline }}\right)>0\right\}\right]$.
} 


\section{Subproblem C: Uncertainty Propagation}

This subproblem aims at finding the ranges of the metrics $J_{1}$ and $J_{2}$ in Equations (8) and (10) that result from propagating both the original uncertainty model and a reduced one. The reduced model, in which the respondents chose 4 out of the 17 category II and III parameters to be improved, was provided by NASA. This is a practical limitation that may stem from having a limited amount of time or money to generate better uncertainty models. In particular, the tasks of interest are as follows:

C1) Find the range of $J_{1}$ corresponding to an uncertainty model based on your response to A3 and the information in Table 2.

C2) Find the range of $J_{2}$ corresponding to an uncertainty model based on your response to A3 and the information in Table 2.

C3) Select 4 category II-III parameters out of the 17 available according to the rankings in B2 and B3, and request from us an improved uncertainty model for them. While improved models for any four parameters will likely lead to smaller ranges of variation, the set of 4 leading to the smallest ranges is ideal. Each working group may request a reduced uncertainty model of 4 parameters of their choice. Only one set of reduced parameters will be provided to each working group.

C4) Use the reduced uncertainty model to recalculate the ranges of $J_{1}$ and $J_{2}$.

A cautionary note on the approaches used to calculate the ranges of $J_{1}$ and $J_{2}$ is in order. Methods to calculate these ranges may lead to underpredictions or overpredictions of the actual range. Each of these two outcomes has its own drawbacks. An underprediction (e.g., a situation where the search for the end points of the range fails to converge to a global optima) can lead the decision maker to the wrong decision (e.g., the estimate of largest failure probability is half of the actual value). An overprediction (e.g., a situation resulting from replacing a distributional p-box with a free p-box) can not only lead the decision maker to the wrong decision (e.g., the estimate of largest failure probability is twice the actual value) but also prevent him/her from making any decision (e.g., the predicted range of failure probabilities covers the entire $[0,1]$ interval).

\section{Subproblem D: Extreme Case Analysis}

This subproblem aims at identifying the epistemic realizations that prescribes the extreme values of $J_{1}$ and $J_{2}$. In particular, the respondents were challenged to do the following tasks.

D1) Find the epistemic realizations of the category II and III parameters that yield the smallest and the largest value of $J_{1}$ for the original uncertainty model used in $\mathrm{C} 1$. Do the same for the reduced uncertainty model used in $\mathrm{C} 4$.

D2) Find the epistemic realizations of the category II and III parameters that yield the smallest and the largest value of $J_{2}$ for the original uncertainty model used in $\mathrm{C} 2$. Do the same for the reduced uncertainty model used in $\mathrm{C} 4$. 
D3) Identify a few representative realizations of $\boldsymbol{x}$ leading to $J_{2}>0$. These realizations should typify different failure scenarios. Describe the corresponding relationships between $\boldsymbol{x}$ and $\boldsymbol{g}$ qualitatively, e.g., the combination of small values of $\boldsymbol{x}_{\mathbf{1}}$ and large values of $\boldsymbol{x}_{\mathbf{2}}$ yields to violations in the $\boldsymbol{g}_{\mathbf{1}}<0$ and $\boldsymbol{g}_{\mathbf{4}}<0$ requirements.

The response to subproblems $\mathrm{C}$ and $\mathrm{D}$ should be in agreement. Note however, that some approaches for addressing subproblem $\mathrm{C}$ are unable to find the extreme-case epistemic realizations sought for here.

\section{Subproblem E: Robust Design}

In this section we consider the multidisciplinary system having the uncertain parameter $\boldsymbol{p} \in \mathbb{R}^{21}$ and the design variable $\boldsymbol{d} \in \mathbb{R}^{14}$ as inputs; and the same $\boldsymbol{g} \in \mathbb{R}^{8}$ used previously as output. The objective of this subproblem is to identify design points $\boldsymbol{d}$ with improved robustness/reliability characteristics. In particular, the respondents were challenged to do the following tasks.

E1) Find a design point $\boldsymbol{d}$ that minimizes largest value of $J_{1}$ for the uncertainty model used in task C4. Provide the resulting value of $\boldsymbol{d}$ and the corresponding range of expected values. In regard to the range of $J_{1}$, is the resulting design better than $\boldsymbol{d}_{\text {baseline }}$ ?

E2) Find a design point $\boldsymbol{d}$ that minimizes the largest value of $J_{2}$ for the uncertainty model used in task C4. Provide the resulting value of $\boldsymbol{d}$ and the corresponding range of failure probabilities. In regard to the range of $J_{2}$, determine if the resulting design is better than $\boldsymbol{d}_{\text {baseline? }}$ ?

E3) Apply the sensitivity analysis of task B2 to the design point found in E1, and the sensitivity analysis of task B3 to the design point found in E2. Compare the rankings with those for the baseline design performed previously.

\section{The Physical System}

The mathematical model $\mathcal{S}$ describes the dynamics of the Generic Transport Model (GTM), a remotely operated twin-jet aircraft developed by NASA Langley Research Center. Figure 1 shows the flight test article and its concept of operations. The aircraft is piloted from a ground station via radio frequency links by using on-board cameras and synthetic vision technology. The parameters in $\boldsymbol{p}$ are used to describe losses in control effectiveness and time delays resulting from telemetry and communications as well as to model a spectrum of flying conditions that extend beyond the normal flying envelope. The requirements in $\boldsymbol{g}$ are used to describe the vehicles stability and performance characteristics in regard to pilot command tracking and handling/riding qualities. Additional information is available in $[4,5,6,7]$. The "black box" format of the formulation of this challenge problem aims at making the problem amenable to the largest possible audience without favoring or hindering respondents depending upon their particular field of expertise. 

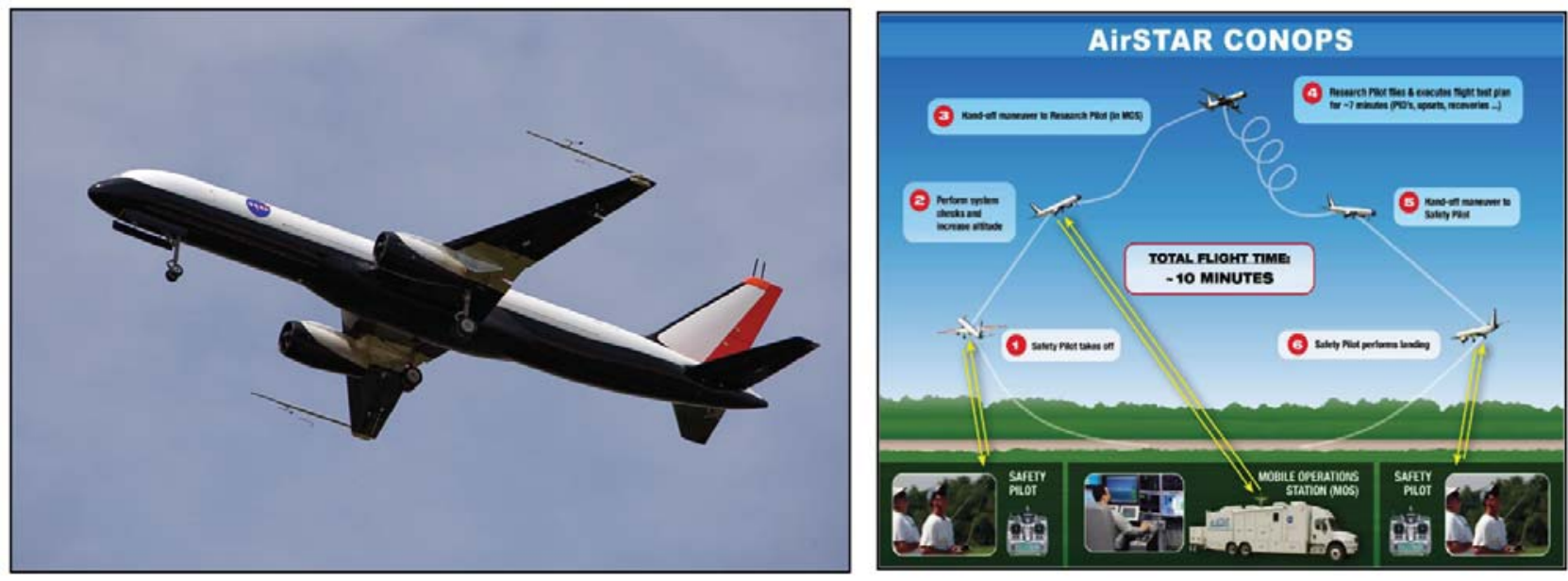

Figure 1. NASA GTM test article and its concept of operations.

\section{Software}

The MATLAB ${ }^{\circledR}$ scripts associated with all five subproblems can be downloaded from http://uqtools.larc.nasa.gov/nda-uq-challenge-problem-2014/. These scripts require the Control System Toolbox to run. The m-files and datafiles required to study each of the subproblems are as follows:

- Subproblem A: p_to_x1.m, x1samples1.mat and x1samples2.mat.

- Subproblems B, C, and D: p_to_x.m and x_to_g.m.

- Subproblem E: p_to_x.m and x_and_d_to_g.m.

The function montecarlo.m, which is not associated with any subproblem in particular, carries out a Monte Carlo simulation of $\boldsymbol{g}$. Details on the inputs and outputs of these scripts can be found by looking at internal comments.

\section{References}

${ }^{1}$ Oberkampf, W., Helton, J. C., Joslyn, C. A., Wojtkiewicz, S. F., and Ferson, S., "Challenge problems: uncertainty in system response given uncertain parameters." Reliability Engineering and System Safety, Vol. 85, 2004, pp. 11-19.

${ }^{2}$ Roy, C. and Oberkampf, W., "A Comprenhensive Framework for Verification, Validation, and Uncertainty Quantification in Scientific Computing," Computer methods in applied mechanics and engineering, Vol. 200, 2011, pp. 2131-2144.

${ }^{3}$ Ferson, S., Kreinovich, V., Ginzburg, L., Myers, D. S., and Sentz, K., "Constructing Probability Boxes and Dempster-Shafer Structures," Tech. Rep. SAND2002-4015, Sandia National Laboratories, 2003.

${ }^{4}$ Jordan, T. L., Foster, J. V., Bailey, R. M., and Belcastro, C. M., "AirSTAR: A UAV Platform for Flight Dynamics and Control System Testing," AIAA Guidance Navigation and Control Conference, Keystone, Colorado, USA, August 2006, AIAA-2006-3307.

${ }^{5}$ Murch, A. M., "A Flight Control System Architecture for the NASA AirSTAR Flight Test Facility," AIAA Guidance Navigation and Control Conference, Honolulu, Hawaii, USA, August 2008, AIAA-20086990. 
${ }^{6}$ Cunningham, K., amd D. G. Murri, D. E. C., and Riddick, S. E., "A Piloted Evaluation of Damage Accommodating Flight Control Using a Remotely Piloted Vehicle," AIAA Guidance Navigation and Control Conference, Portland, Oregon, USA, August 2011, AIAA-2011-6451.

${ }^{7}$ Crespo, L. G., Matsutani, M., and Annaswamy, A., "Design of an adaptive controller for a remotely operated air-vehicle," AIAA Journal of Guidance, Control and Dynamics, Vol. 35, No. 2, 2012, pp. 406-422. 